\title{
Principles of Psychosocial Programming for Children and Cancer
}

\author{
Mark A. Chesler, Ph.D. (Co-Chairperson), ${ }^{*}$ Sue P. Heiney, M.N., R.N., C.S. (Co- \\ Chairperson), $\uparrow$ Rosemarie Perrin (Rapporteur), $\ddagger$ Grace Powers Monaco, J.D., $\S$ \\ Mary Jo Kupst, Ph.D., I Nancy F. Cincotta, M.S.W., Ernest R. Katz, Ph.D.,\# \\ Patricia Deasy-Spinetta, M.S., N.C.S.P., ${ }^{* *}$ Elizabeth H. Whittam, R.N., F.N.P.,†† \\ and Genevieve V. Foley, R.N., M.S.N., O.C.N., C.N.A.A.††
}

The working group on psychosocial issues believes that we need a protocol or checklist, along with tested examples, to guide local practice, research, and assessment of institutional psychosocial services and support for children, teenagers, and families affected by cancer. Such a protocol should be standardized, but it should be designed to accommodate the individual needs of both the patient and family and of the caregivers and their institutions. It also should have as its objective the normalization and empowerment of the child and the family. The protocol must be comprehensive, and it must take a longitudinal approach to care. Above all, it should be accessible and feasible. Such a protocol, once developed, should lend itself to funding support from a variety of community and national organizations.

Development of such a protocol is beyond the scope of this working group in this limited time frame. As noted at the conclusion of this report, we recommend establishment of a special Psychosocial Task Force to take the next steps and develop such a protocol. However, we have identified here the key princi-

Presented at the American Cancer Society Workshop on Children with Cancer, Naples, Florida, September 5-6, 1991.

From the ${ }^{*}$ University of Michigan, Ann Arbor, Michigan; the †Children's Center for Cancer and Blood Disorders of Richland Memorial Hospital, Columbia, South Carolina; the $\ddagger$ American Cancer Society; the $\S$ Candlelighters Childhood Cancer Foundation, Washington, DC; the \|Midwest Children's Cancer Center, College of Wisconsin, Milwaukee, Wisconsin; the IDepartment of Social Work Services, Mount Sinai Hospital, New York, New York; the \#Jacques Children's Cancer Center, Long Beach Memorial Miller Children's Hospital, Long Beach, California; the **San Diego Unified School District, San Diego, California; and the $† \dagger$ Memorial Sloan-Kettering Cancer Center, New York, New York.

Address for reprints: Mark A. Chesler, Ph.D., University of Michigan, 4016 LAS Building, Ann Arbor, Ml 48109-1382.

Accepted for publication May 18, 1992. ples on which this protocol can be constructed, at the same time enumerating potential impediments to implementation of each principle. These follow.

1. Psychosocial care is, and should be seen as, essential to improving the chances for disease-free and life-enhancing futures for everyone affected by childhood cancer. It is fundamental to the promotion of physical and mental health and social productivity.

Impediments:

- Many providers do not fully believe this or understand its full ramifications, or do not have the time, energy, or skill to act on it

- Some parents see such services as "extras," invasions of privacy, or as signs of weakness

- There is a lack of reimbursement mechanisms for psychosocial services

2. A comprehensive psychosocial program should promote positive emotional growth and development and the maintenance of normal childhood, adolescence, and young adulthood.

Impediments:

- Complexity and compartmentalization of the medical system and services and its distance from parent and community organizations

- Preexisting psychosocial problems in the child or family

- Inadequate staffing

- Complexity of such a program

- Societal prejudice and discrimination

3. A psychosocial care program should focus on the child and family, with attention to how the family defines itself, its support network, and its stage in the life cycle. 
Impediments:

- Outdated perceptions of what constitutes the family

- Need for a wide range of diversified services and a staff knowledgeable about individual and cultural differences

4. The program should focus on coordination and use of the wide range of psychosocial services and partners available in the community-schools, churches, peer groups, parent groups, parent support groups (such as Candlelighters), financial institutions, employers, and community organizations.

Impediments:

- Concern about duplication of community services

- Ignorance of community resources

- Turf battles

- Lack of staff and family networking skills and lack of advocacy of parent groups

- Failure to recognize nontraditional resources for provision of psychosocial services

5. All children and families should have access to respectful and culturally sensitive psychosocial programs.

Impediments:

- Current lack of access, especially by racially, economically, and ethnically disadvantaged groups

- Financial impediments

- Staff inflexibility to and lack of knowledge of changing ethnic needs

- Low recruitment of racial and ethnic minorities into psychosocial professions

- Lack of adequate knowledge base from which to build such programs

- Current lack of health care team interface with other community agencies that provide services to minority and ethnic groups and, thus, underutilization of their resources

6. Inherent in a psychosocial program is commitment to longitudinal support, from prediagnosis and throughout life, especially to meet needs reflected in recent research dealing with late effects, bereavement, genetics, and long-term survivors.

Impediments:

- Narrow definitions of care and period of need

- Lack of appropriate partnerships (e.g., between Candlelighters and professionals) and lack of resources that could facilitate lifetime follow-up
7. Models of psychosocial care should be based on basic and clinical behavioral science research (both research on the childhood cancer experience and on other disease and life situations), clinical expertise, and patient/family experiences.

Impediments:

- Failure to disseminate results of current effective models

- Local difficulties in implementation (i.e., financial, expertise)

- Lack of cooperative clinical trials and methods to incorporate such basic and intervention-focused psychosocial research

- Failure to recognize, use, and integrate research and expertise from other chronic illnesses and intervention situations

- Lack of cooperation between professionals and established groups with expertise in family and youth issues (such as Candlelighters)

- Underrecognition of the American Cancer Society as a resource for funding psychosocial research; the Society should continue efforts to promote availability of research grants to a broad, multidisciplinary audience via journals reaching such professionals

8. Efforts must be made to involve new partners in the generation of funds to support psychosocial programs, including psychosocial providers and families lobbying third-party payers to reimburse for mental health services. In addition, partnerships with community groups and Candlelighters parent groups can help raise funds for psychosocial care and research.

Impediment:

- Lack of staff and parent experience in creating and maintaining innovative partnerships

9. Each member of the formal or informal team, including professionals, parents, and patients, contributes in an important way to psychosocial support. These contributions are based most effectively on an understanding of each individual's role, respect for their unique contribution, and willingness to communicate openly about psychosocial issues.

Impediments:

- Personal/cultural values may contribute to discomfort or inability to participate in such a program of psychosocial care

- Knowledge deficits and specialized professional jargons

- Staff and/or patient/family willingness and ability 
to engage in open and mutually respectful interaction

- Status differences and hierarchies

- Failure to incorporate into medical education the concepts of psychosocial care and research

10. Children and adolescents diagnosed with cancer must receive care at cancer centers with full programs of psychosocial assessment, intervention, and support for themselves and their families. These programs should be directed and implemented by staff with particular expertise in psychosocial care.

Impediments:

- Lack of people, resources, and institutional priority

- Inadequate links to local providers in communities distant from a cancer center

11. Joining forces with groups and medical specialties serving children with other diseases or life situations can increase our wisdom, foster the sharing of ideas and strategies, strengthen advocacy, improve access to funding, and export our paradigm of psychosocial care.

Impediments:

- Fear on the part of individuals and organizations that dilution or loss of identity and resource base will occur

- Territoriality

- Belief that cancer is so special that we cannot learn much from other childhood diseases or family crises

12. The unique perspectives of the child/adolescent and family regarding their emotional and social needs should be incorporated into all components of the psychosocial and medical program.

Impediments:

- Caregiver and family attitudes that minimize the importance of an active child and family role in care

- Professional discomfort in sharing control over medical and psychosocial treatment

- Lack of staff, family, and patient skills in listening and communicating

13. Comprehensive psychosocial programs need to be multifaceted, with ample opportunities for children and families to be supported throughout the course of illness, treatment, survivorship, and bereavement. Impediments:

\section{- As above in 1-12}

14. Ongoing support for caregivers across disciplines is important in adapting to the stresses inherent in working with children with cancer. In particular, peer support and education and training can contribute to the comfort of health care professionals in addressing psychosocial issues.

Impediments:

- Not institutionally sanctioned

- Not a priority of health care professionals

- Lack of adequate research on effective modes of staff support, education, and reeducation

The working group on Psychosocial Issues recommends that the American Cancer Society appoint a special Psychosocial Task Force to follow up on these guiding principles to develop a protocol or checklist, along with practical and tested examples, to guide local practice, research, education, and assessment of care. If a task force is not forthcoming, then a consortium of organizations involved in psychosocial care (i.e., Candlelighters, the Association of Pediatric Oncology Nurses, the Association of Pediatric Oncology Social Workers, the Association of Pediatric Psychologists, and other pertinent organizations) should be convened and supported to accomplish this task.

Such a protocol provides a method to assess both family needs and institutional services. Additionally, the protocol would delineate the steps for effective use of psychosocial services by institutions. Initially, the protocol would address needs at diagnosis, but eventually would be extended to all phases of the cancer trajectory. A component in this protocol would be a method to evaluate the impact of these services. Our group has gathered and integrated some of these examples from research and practice, but a larger commitment of time and other perspectives is required to develop such a detailed protocol or checklist and concrete practical strategies for their dissemination and implementation. An overall recommendation is that the task force on psychosocial issues address the impediments and develop specific ways to overcome the obstacles identified. 